EDITOR'S
${ }^{1}$ Centre for Anaesthesia, University College London Hospital, London, UK ${ }^{2}$ Surgical Outcomes Research Centre (SOuRCe) UCL/UCLH Joint Comprehensive Biomedical Research Centre, Department of Anaesthetics, University College London Hospital, London, UK

\section{Correspondence to}

Dr Sarah Barnett, Centre for Anaesthesia, 3rd Floor Maples Link Corridor, University College London Hospital, 235 Euston Road, London NW1 2BU, UK; sarahfbarnett@googlemail.com

Received 4 October 2010 Accepted 7 December 2010 Published Online First 21 January 2011

\title{
Clinical risk scores to guide perioperative management
}

\author{
Sarah Barnett, ${ }^{1}$ Suneetha Ramani Moonesinghe ${ }^{2}$
}

\section{ABSTRACT}

Perioperative morbidity is associated with reduced long term survival. Comorbid disease, cardiovascular illness, and functional capacity can predispose patients to adverse surgical outcomes. Accurate risk stratification would facilitate informed patient consent and identify those individuals who may benefit from specific perioperative interventions. The ideal clinical risk scoring system would be objective, accurate, economical, simple to perform, based entirely on information available preoperatively, and suitable for patients undergoing both elective and emergency surgery. The POSSUM (Physiological and Operative Severity Score for the enUmeration of Mortality and Morbidity) scoring systems are the most widely validated perioperative risk predictors currently utilised; however, their inclusion of intra- and postoperative variables precludes validation for preoperative risk prediction. The Charlson Index has the advantage of consisting exclusively of preoperative variables; however, its validity varies in different patient cohorts. Risk models predicting cardiac morbidity have been extensively studied, despite the relatively uncommon occurrence of postoperative cardiac events. Probably the most widely used cardiac risk score is the Lee Revised Cardiac Risk Index, although it has limited validity in some patient populations and for non-cardiac outcomes. Bespoke clinical scoring systems responding to dynamic changes in population characteristics over time, such as those developed by the American College of Surgeons National Surgical Quality Improvement Program, are more precise, but require considerable resources to implement. The combination of objective clinical variables with information from novel techniques such as cardiopulmonary exercise testing and biomarker assays, may improve the predictive precision of clinical risk scores used to guide perioperative management.

\section{INTRODUCTION}

Perioperative morbidity and mortality is a significant public health issue, due to its impact on patients' short and long term survival, and also resource utilisation within the health service. Surgical complications occur in $3-17 \%$ of patients ${ }^{1}{ }^{2}$; in the general population, surgical mortality is approximately $0.5 \%$, but in elderly patients undergoing emergency surgery the UK mortality rate is $>12 \%{ }^{3}$

As clinicians we strive to find accurate methods to stratify patient risk from undergoing surgery. Clinical risk scores tend to focus on patient history, examination, preoperative investigations, surgical severity, and intraoperative events. Surgical risk may also be assessed using measures of functional capacity, such as the Duke Activity Status Index, and more objectively, using cardiopulmonary exercise testing (CPET). ${ }^{4} 5$ There is also increasing interest in biological markers of cardiorespiratory health or inflammation which could be used to predict perioperative outcome such as $\mathrm{N}$-terminal brain natriuretic peptide (NT-BNP) ${ }^{6}$ and high sensitivity $\mathrm{C}$ reactive protein (hsCRP). ${ }^{8}{ }^{9}$ However, while both CPET and serum biomarkers show promise as risk prediction tools, neither are widely available, and both require further validation in large pragmatic multicentre studies.

Scoring systems have the advantage that they are usually cost neutral, and can be performed at any time of the day or night. Patients presenting for emergency surgery are at high perioperative risk, ${ }^{3}$ and it may be possible to predict the outcomes of such patients using these openly available, relatively quick scoring systems which are not subject to complex interpretation.

The ideal risk prediction model would be one that is simple, reproducible, accurate, objective, and available to all patients. Many hospitals lack the resources to run expensive tests, so ideally it should be cheap, and possible to perform at the bedside. As the purpose of the scoring system is to define an individual patient's risk before he or she undergoes surgery, a model that is based entirely on preoperative risk factors would be more beneficial than those which include intraoperative and postoperative variables.

In the course of this article we shall describe the different scoring systems that have been developed and are used to predict risk for non-cardiac surgery, highlighting their advantages and disadvantages, and how their accuracy has be shown to vary in a number of different studies.

\section{RISK STRATIFICATION SCORING SYSTEMS}

Risk stratification scoring systems can be classified into those estimating population risk, such as the American Society of Anaesthesiologists Physical Status score (ASA-PS), and those estimating individual risk. Risk scores developed to calculate individual risk can be subdivided into those which are designed to predict cardiac morbidity and mortality such as the Lee Revised Cardiac Risk Index (RCRI), and those which predict generic morbidity and mortality. These include scores which use solely preoperative risk factors-for example, Charlson Index-and those looking at a combination of preoperative, intraoperative, and postoperative factors-for example, Physiological and Operative Severity Score for the enUmeration of Morbidity and Mortality (POSSUM). We shall now consider each of these in turn. 
POPULATION RISK: AMERICAN SOCIETY OF ANAESTHESIOLOGISTS' PHYSICAL STATUS SCORE

The ASA-PS uses preoperative physical fitness to categorise patients subjectively into five subgroups. The score was devised in 1941 originally as a statistical tool for retrospective analysis of hospital records. ${ }^{10}$ It has been revised on several occasions and in 1963 the five point scale most commonly known was first reported. ${ }^{11}$ There is now an additional category for patients whose organs are being removed for donor purposes (table 1).

Underlying fitness is an important predictor of survival from surgery, and the ASA-PS score has been shown to correlate with outcome in a number of different settings. ${ }^{12-23}$ It is simple, easy to understand, and commonly used as part of the preoperative assessment. It is also an easy and useful tool to assist descriptions of workload and 'anaesthetic risk' for audit and research purposes.

However, the ASA-PS system has a number of limitations. It does not consider the preoperative optimisation of the patient, the surgery planned, or the proposed postoperative care-that is, critical care unit admission-and it makes no adjustments for age, sex, weight, or pregnancy. In this sense it does not give a prediction of risk for an individual patient or operation. Wolters' study found a population mortality of $0.1 \%$ for ASA I patients, and an $18.3 \%$ mortality for patients graded ASA IV. ${ }^{24}$ However, more recent work demonstrated that the ASA-PS was poorly predictive of individual patient outcome: while it correctly predicted an uncomplicated course in $96 \%$ of patients, complications were correctly predicted in only $16 \%$ of patients in whom they occurred (positive predictive value $57 \%$, negative predictive value $80 \%) .{ }^{25}$ Similar results were found in a more recent study which looked at infective complications and comorbidity in patients undergoing total knee replacement. ${ }^{26}$

\section{INDIVIDUAL RISK: CARDIOVASCULAR MORBIDITY AND MORTALITY}

In 1977 Goldman et al developed a cardiac risk index using nine different preoperative variables. ${ }^{27}$ Since then, multiple cardiac risk indices have been developed, and there have been a number of guidelines which recommend their use for preoperative cardiac evaluation. ${ }^{28} 29$

Probably the most widely used cardiac risk index currently was developed by Lee et al in 1999, by revising Goldman's criteria for cardiac risk and using six independent variables. ${ }^{30} \mathrm{~A}$ patient is considered to be high risk when they have more than two risk factors. A recent systematic review evaluated the ability of the revised cardiac risk index (RCRI) to predict cardiac complications and mortality after major non-cardiac surgery across different populations and settings. ${ }^{31}$ Their conclusion was the RCRI was moderately good at discriminating patients who developed complications from those whose clinical course was uneventful after mixed (non-vascular and vascular) non-cardiac surgery. It was less accurate at discriminating cardiac events

Table 1 American Society of Anaesthesiologists Physical Status Score ${ }^{11}$

\begin{tabular}{ll}
\hline ASA grade \\
\hline I & A normal healthy patient \\
II & A patient with mild systemic disease \\
III & A patient with severe systemic disease \\
IV & A patient with severe systemic disease that is a constant threat to life \\
V & A moribund patient who is not expected to survive without the operation \\
VI & A declared brain dead patient whose organs are being removed for donor \\
& purposes
\end{tabular}

after vascular non-cardiac surgery and less accurate at predicting mortality. Acknowledged limitations of the review included the statistical and clinical heterogeneity and generally low methodological quality of the included studies, and their varied definitions of "cardiac events" (box 1).

There is ongoing work on adapting and improving the predictive accuracy of the RCRI. A recent prospective, single centre study assessed the predictive power of NT-proBNP, CRP, and RCRI in combination for the risk of a perioperative major cardiovascular event, and found that the predictive power of the RCRI could be improved significantly by the addition of CRP and NT-proBNP to RCRI (adjusted relative risk (RR) 4.6) $(p<0.001){ }^{32}$

While cardiac morbidity is important, it is relatively uncommon compared with other types of perioperative complication, which may have significant implications for patients both in the short and long term. ${ }^{33} 34$ There is therefore a requirement for a clinical risk score which may predict generic morbidity and mortality with accuracy. Existing systems which are used for this purpose are now discussed.

\section{GENERIC MORBIDITY AND MORTALITY: PREOPERATIVE RISK FACTORS ALONE}

The Charlson Index was developed in 1987 and was based on the 1 year mortality data from 604 medical patients admitted to a New York hospital. The index was then validated for its ability to predict death in a cohort of 685 breast cancer patients. ${ }^{35}$ The authors went on to validate an age-comorbidity index (Charlson Age Comorbidity Index, CACI) in a non-cardiac cohort of patients to predict long term outcome. Two hundred and twenty-six patients undergoing elective surgery were enrolled in the study, all of whom had hypertension or diabetes. These patients were followed for at least 5 years postoperatively. The estimated RR of death for each comorbidity rank was 1.4 and for each decade of age was 1.4. When age and comorbidity were represented as a combined score the estimated RR for each combined unit was 1.45. The estimated RR of death from an increase of one in the comorbidity score was approximately equal to that from an additional decade of age ${ }^{36}$ (table 2 ).

It has since been validated for predicting inpatient morbidity and mortality in a number of different surgical cohorts. A study of lung cancer patients ${ }^{37}$ identified gender, CACI score 3-4, chronic obstructive pulmonary disease (COPD), and prior tumour within the last 5 years as predictors for major complications. Charlson scores of 3-4 maintained statistical significance in multivariate regression analyses (OR 9.8, 95\% CI 2.1 to 45.9). Ouellette et al also found the CACI to be a predictor of inhospital morbidity, duration of hospital stay and mortality

\section{Box 1 Revised Cardiac Risk Index (Lee et al 1999) ${ }^{30}$}

1. High risk type of surgery

2. Ischaemic heart disease (includes any of the following: history of myocardial infarction, history of a positive exercise test, current complaint of chest pain, ie, considered to be secondary to myocardial ischaemia, use of nitrate therapy, or electrocardiography with pathologic 0 waves)

3. Congestive heart failure

4. History of cerebrovascular disease

5. Preoperative treatment with insulin

6 . Preoperative serum creatinine $>2.0 \mathrm{mg} / \mathrm{dl}$ 
Table 2 Charlson Index (Charlson et al 1987) $)^{35}$

\begin{tabular}{lll}
\hline $\mathbf{1}$ & $\mathbf{2}$ & $\mathbf{3}$ \\
\hline Myocardial infarction & Hemiplegia & $\begin{array}{l}\text { Moderate or severe liver disease } \\
\text { (eg, cirrhosis with ascites) }\end{array}$ \\
$\begin{array}{l}\text { Congestive heart failure } \\
\text { Peripheral vascular disease }\end{array}$ & $\begin{array}{l}\text { Moderate or severe renal disease } \\
\text { Diabetes with end organ damage } \\
\text { Cerebrovascular disease }\end{array}$ & Any malignancy \\
Dementia & \\
Chronic pulmonary disease & \\
Connective tissue disease & \\
Ulcer disease & \\
Mild liver disease & \\
Diabetes & \\
\hline
\end{tabular}

following colorectal surgery, ${ }^{38}$ and it has helped to calculate mortality following cardiac surgery. ${ }^{39}$ The CACI has also, in certain patient groups, been found to predict 'long term' mortality. 40

Following head and neck surgery, ${ }^{21}$ and radical prostatectomy, ${ }^{42}$ the Charlson Index has a predictive ability comparable to that of the ASA-PS when ascertaining morbidity and mortality. However, the ASA-PS was more accurate in its calculation than the Charlson Index in a cohort of patients undergoing liver resection. ${ }^{43}$

This series of papers represents a relatively large and diverse experience with the Charlson Index, mainly confirming its predictive validity. However, disadvantages of this scoring system are the lack of information regarding the surgical procedure, and the subjectivity of the patients' comorbidity assessment, which may potentially lead to error.

\section{GENERIC MORBIDITY AND MORTALITY: PERIOPERATIVE RISK FACTORS}

The Physiological and Operative Severity Score for the enUmeration of Mortality and Morbidity (POSSUM) was developed by Copeland et al in 1991 as a scoring system for surgical audit, ${ }^{44}$ where they used multivariate logistic regression to identify the most significant of 48 physiological variables and 12 operative and postoperative variables for the prediction of the 30 day morbidity and mortality rates. The final POSSUM system incorporates 12 variables to assess physiological status and six 'surgical' variables, resulting in an 18 component score. In order for perioperative risk to be calculated, the sum of the physiological and surgical variables are entered into two mathematical equations which are used to calculate the risk of morbidity and mortality (table 3).

A potential drawback in using this scoring system to assess risk is the time at which the operative variables are obtained. The physiological variables are documented before the commencement of surgery, and include the patient's clinical symptoms, signs elicited, haematology and biochemistry results, and an assessment of the electrocardiogram. When it comes to collecting the surgical data, this can be delayed for a prolonged period of time, as included in the score are the number of subsequent operations within 30 days, and presence of malignancy. This causes obvious difficulties in using this as the sole tool to make decisions on the appropriateness of surgery, as a number of variables will not be available until the surgery is complete.

POSSUM was originally developed and validated in a single UK district general hospital, in a study which included emergency and elective procedures in patients undergoing urological, gastrointestinal, vascular, and hepatobiliary surgery. ${ }^{44}$ Subsequently, this system has also been used in comparisons of surgeons, ${ }^{45}$ resource utilisation, ${ }^{46}$ and to compare surgical outcomes in different countries. ${ }^{47}$ While some studies have found confirmatory evidence that POSSUM predicts individual patient risk of morbidity and mortality, ${ }^{48-50}$ others have found a significant overestimation of mortality using this score. ${ }^{51-57}$ This is the result of using logistic regression to predict risk, as the lowest possible mortality risk is $1.08 \%{ }^{58}$

Using alternative risk equations, but the same physiological and surgical variables, a new risk model was developed in Portsmouth (P-POSSUM), and validated in a large single centre cohort, ${ }^{59}$ and since then has been shown to predict in-hospital mortality more accurately than POSSUM.48 5657 60-64 However, P-POSSUM has no morbidity prediction equation, as a result of the original authors' lack of confidence in the reporting of perioperative complications. ${ }^{59}$ Subsequent studies have shown P-POSSUM to both over-predict ${ }^{54}{ }^{64}$ and under-predict mortality 486165 in different settings.

Large cohorts of patients have been used to validate variations of POSSUM for specific surgical groups, including Cr-POSSUM (colorectal surgery) ${ }^{54} \quad 63 \quad 65 \quad 66$ and V-POSSUM (vascular surgery), ${ }^{50}$ which are more sensitive and specific for predicting patient outcome following these operations. However, a recent systematic review of the use of different POSSUM models in oesophageal-gastric surgery found that the P-POSSUM was superior to both the original POSSUM and the surgery specific O-POSSUM model for the prediction of postoperative mortality. ${ }^{67}$ These data highlight the considerable variation in the predictive precision of these models in different surgical cohorts. One of the reasons for this variation may be the inclusion of a number of subjective variables such as jugular venous pressure measurement and chest radiograph interpretation, which may introduce error in risk score calculation. Nevertheless, POSSUM and its subsequent variations remain the most internationally validated risk scoring system for predicting individual patient risk.

\section{BIOCHEMICAL AND HAEMATOLOGICAL OUTCOME MODELS}

The Biochemistry and Haematology Outcome Model (BHOM) was developed by Prytherch et al ${ }^{68}$ in 2003, and data collected includes age, sex, mode of admission (eg, emergency or elective), physiological parameters (haemoglobin, white cell count, sodium, potassium, urea), BUPA operative severity score, and 30 day mortality. The BUPA operative severity score is used in the private sector (UK) as an indicator of surgical complexity and workload. The BHOM uses information which is easily obtainable from most hospital information technology systems to calculate predictive mortalities. ${ }^{69} 70$ As previously stated, a risk assessment scoring system should ideally have few variables, which are simple to collate and therefore make the score 
Table 3 Physiological and Operative Severity Score for the enUmeration of Mortality and Morbidity (POSSUM) (Copeland et al) ${ }^{44}$

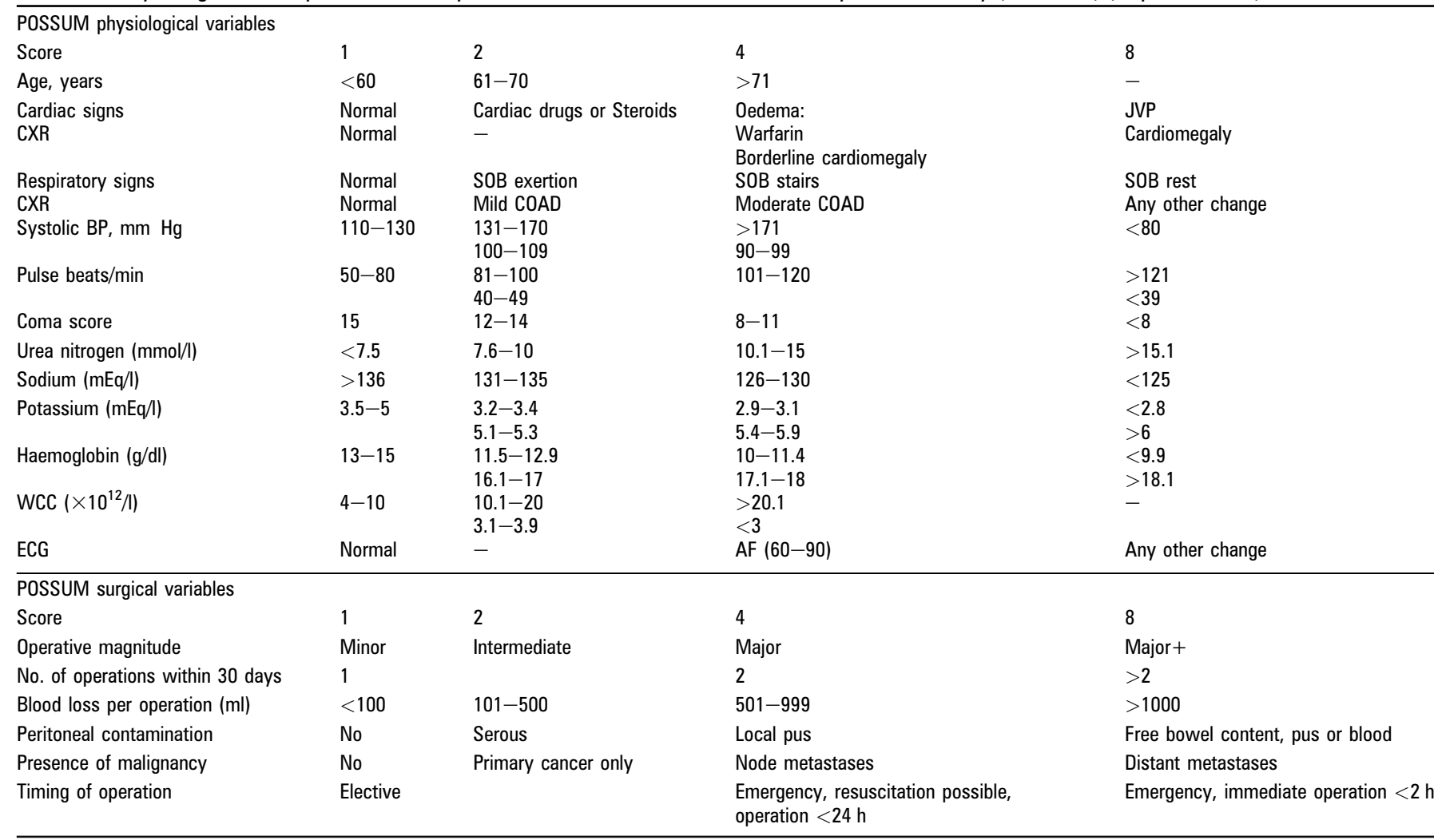

AF, atrial fibrillation; COAD, chronic obstructive airway disease; CXR, chest x ray; BP, blood pressure; ECG, electrocardiogram; JVP, jugular venous pressure; SOB, shortness of breath; WCC white cell count.

easy to implement in daily practice. It should be available for every patient and have limited potential inter-observer variability. BHOM uses objective data, which are easily available from a single blood sample. It is therefore feasible to collect this for all patients as part of routine care. However, BHOM is one of the less widespread outcome models used presently, and is yet to be validated in large multicentre cohorts including patients undergoing different types of surgery.

\section{THE SURGICAL APGAR SCORE}

The Apgar score was originally developed as a powerful tool in obstetrics to rapidly assess and gain feedback on a newborn's condition. ${ }^{71}$ The surgical Apgar score was piloted using general and vascular patients where the score was significantly associated with the occurrence of major complications or death within 30 days of surgery $(p<0.001) .^{72}$ The 10 point score is calculated from the estimated blood loss, lowest heart rate, and lowest mean arterial pressure during an operation. It uses routinely available data, and has been to shown to identify immediately and effectively those patients at higher and lower risk of complications and death postoperatively. ${ }^{73}$ Its limitations include its lack of validity in other cohorts of patients, lack of comparison between different institutions, and the potential for imprecision resulting from 'estimating' blood loss. As a consequence, it may be considered one of the less applicable scoring systems in current clinical practice.

\section{AN AMERICAN APPROACH: THE NATIONAL SURGICAL QUALITY IMPROVEMENT PROGRAM}

The American College of Surgeons National Surgical Quality Improvement Program (ACS NSOIP) is the first nationally validated, risk adjusted, outcomes based programme used to measure and improve the quality of surgical care in the USA. ${ }^{74} \mathrm{~A}$ prospective, validated database is used to quantify 30 day risk adjusted surgical outcomes. A comparison of outcomes can then be made for all the hospitals within the programme. ACS NSOIP collects data on 136 variables for patients undergoing major surgical procedures, including preoperative risk factors, intraoperative variables, and 30 day postoperative morbidity and mortality. The data are collected, validated, and submitted by a trained surgical clinical reviewer at each site. Using logistic regression, risk adjusted 30 day morbidity and mortality outcomes are made for each participating hospital on an annual basis. Both generic and surgery specific models are generated, and outcomes are reported as observed versus expected $(\mathrm{O} / \mathrm{E})$ ratios. The ACS NSOIP database therefore allows hospital specific adjustments to surgical risk to be incorporated, by allowing the categorisation of hospitals performing better or worse than expected.

The unique aspect of this initiative is the generation of bespoke models which are responsive to changes in population characteristics over time, and therefore are more precise. This was demonstrated in a recent study which compared an ACSNSOIP model for colorectal surgery with existing validated models including the CR-POSSUM and the Association Francaise de Chirugie colorectal model. The ACS NSOIP colorectal risk calculator demonstrated far higher predictive precision than existing models in both development and validation cohorts. ${ }^{75}$ Despite the advantages of such initiatives, their application internationally is limited by the resources required to collect and record multiple variables prospectively and accurately.

\section{CONCLUSIONS AND FUTURE WORK}

Clinicians within the perioperative team utilise risk prediction scores to help make important, informed decisions to optimise 


\section{Main messages}

- An ideal clinical risk score would consist of objective preoperative variables, be economical and simple to implement, and suitable for patients undergoing elective and emergency surgery.

- The POSSUM scoring systems are the most widely validated, internationally available clinical risk scores currently available for the prediction of generic perioperative morbidity and mortality; however, they are not validated for preoperative risk stratification, as they incorporate intraoperative variables. The Lee RCRI is the most widely validated system for the prediction of adverse cardiac outcomes, although it should be recognised that cardiac events account for only a very small fraction of postoperative morbidity, and the RCRI is poorly predictive of all cause morbidity and mortality.

- Initiatives such as the ACS-NSOIP programme in the USA allow the development of bespoke risk prediction models which are responsive to changes in population characteristics and patient management; however, considerable resources would be required to implement such programmes internationally.

an individual's perioperative management. Accurate preoperative determination of risk is also important to enable truly informed consent for patients who may be at high risk, particularly for operations where non-surgical interventions may be an alternative option. Looking at the available research it is clear that there are limitations to many of the risk stratification scoring systems that are currently available.

With clinical risk scores using subjective variables, the method of data collection, and therefore the accuracy of the data collected, may influence the predictive accuracy of a particular risk model. Even relatively simple systems such as the ASA-PS have been demonstrated to be subject to inter-observer variation in their assessment, ${ }^{76}$ and there is a risk of diagnostic inaccuracy with systems which use administrative databases as a source of information, or untrained medical staff as data collectors as opposed to prospective data collection by trained staff. ${ }^{78} 78$ The use of objective variables can improve reliability and precision of risk scoring systems. The technology already available in hospitals may aid future collection of routine variables which can be incorporated into an objective dataset to help predict risk.

\section{Future research}

- Identification of risk factors which are common to the various validated clinical risk scores and use these to develop a simple objective dataset.

- Develop models which incorporate clinical data and information from novel techniques such as cardiopulmonary exercise testing and biomarker assays, to assess if these improve predictive precision.

- Develop large multicentre databases which allow the development and validation of bespoke risk prediction models which may be modified on an annual basis and therefore be responsive to changes in clinical management, patient characteristics, and healthcare delivery.

\section{Key references}

Wolters U, Wolf T, Stutzer $\mathrm{H}$, et al. Risk factors, complications, and outcome in surgery: a multivariate analysis. Eur J Surg 1997;163:563-8.

- Choi J-H, Cho DK, Song Y-B, et al. Preoperative NT-proBNP and CRP predict perioperative major cardiovascular events in non-cardiac surgery. Heart 2010;96:56-62.

- Charlson M, Szatrowski TP, Peterson J, et al. Validation of a combined comorbidity index. J Clin Epidemiol 1994;47:1245-51.

- Dutta S, Horgan PG, McMillan DC. POSSUM and its related models as predictors of postoperative mortality and morbidity in patients undergoing surgery for gastro-oesophageal cancer: a systematic review. World J Surg 2010;34:2076-82.

- Cohen ME, Bilimoria KY, Ko CY, et al. Development of an American College of Surgeons National Surgery Quality Improvement Program: Morbidity and Mortality Risk Calculator for Colorectal Surgery. J Am Coll Surg 2009;208:1009-16.

If this is then combined with biomarker assays and assessment of functional capacity, improved performance may be possible.

Further work is required to improve the identification of patients at high risk of perioperative morbidity. Such work should include both research required to identify objective risk factors which are significant on multivariate analysis and may be combined in a scoring system with high predictive precision, and also investment in resources enabling both objective and subjective variables to be recorded and collected accurately. As medical and surgical practice changes with time, it is likely that the predictive precision of risk scoring systems may also change: the implications of comorbid illness may not be the same now as during the 1990s when systems such as the RCRI and POSSUM score were first validated. Furthermore, standards of healthcare and perioperative practice will differ between institutions and countries, with the consequence that a 'one size fits all' solution may not be possible. Therefore, the ideal risk prediction system, in addition to consisting of predominantly preoperative variables, should also be responsive to changes in clinical management over time. If subjective variables are used, then these should be assessed by staff trained in such assessment. The ACSNSOIP initiative approaches these ideals; however, for the rest of the world, while existing systems such as the RCRI and POSSUM scores are helpful, further work aimed at evaluating and refining them in different populations and healthcare systems is required.

\section{MULTIPLE CHOICE OUESTIONS (TRUE (T)/FALSE (F); ANSWERS AFTER THE REFERENCES)}

\section{The ideal risk prediction model would be}

A. Simple

B. Subjective

C. Expensive

D. Possible to perform at the bedside

E. Purely for elective cases

\section{The American Society of Anaesthesiologists' physical status score \\ A. Is commonly used as part of the preoperative assessment \\ B. Considers the preoperative optimisation of the patient \\ C. Makes no adjustment for the age of the patient}


D. Requires complex calculations

E. Has poor sensitivity and specificity for prediction of individual patients risk of morbidity and mortality

\section{Cardiovascular morbidity and mortality}

A. Is relatively uncommon compared with other types of perioperative complications

B. Goldman's criteria for cardiac risk is the most widely used cardiac risk index

C. The revised cardiac risk index is poor at discriminating cardiac events after mixed (vascular and non-vascular) noncardiac surgery

D. The revised cardiac risk index consists of nine preoperative variables

E. The predictive power of the revised cardiac risk index can be significantly improved by the addition of biological markers

\section{The Physiological and Operative Severity Score for the enumeration of Mortality and Morbidity (POSSUM)}

A. Was first reported to assist comparative audit among anaesthetic services

B. Incorporates 12 physiological variables and six surgical variables

C. Uses purely preoperative variables

D. Contains no subjective variables

E. Is the most internationally validated risk scoring system for predicting individual patient risk

\section{Clinical risk scores}

A. Inter-observer variation in data collection can influence predictive accuracy

B. Adaptation to changes in patient population over time may improve predictive precision

C. The Biochemistry and Haematology Outcome Model (BHOM) has been validated in multicentre cohorts

D. The surgical Apgar score is a 10 point score calculated from intraoperative heart rate, blood pressure, and estimated blood loss

E. The Charlson Age-Comorbidity Index uses no information regarding the surgical procedure

Competing interests SRM works within the University College London/University College London Hospitals' Joint Comprehensive Biomedical Research Centre, which received a proportion of funding from the UK Department of Health's National Institute for Health Research Biomedical Research Centres' funding scheme.

Provenance and peer review Commissioned; externally peer reviewed.

\section{REFERENCES}

1. Kable AK, Gibberd RW, Spigelman AD. Adverse events in surgical patients in Australia. Int J Qual Health Care 2002;14:269-76.

2. Gawande AA, Thomas EJ, Zinner MJ, et al. The incidence and nature of surgical adverse events in Colorado and Utah in 1992. Surgery 1999;126:66-75.

3. Pearse RM, Harrison DA, James P, et al. Identification and characterisation of the high-risk surgical population in the United Kingdom. Critical Care 2006:10:R81.

4. Older $\mathbf{P}$, Hall A, Hader R. Cardiopulmonary exercise testing as a screening test for perioperative management of major surgery in the elderly. Chest 1999:116:355-62.

5. Bolliger CT, Jordan P, Soler M, et al. Exercise capacity as a predictor of postoperative complications in lung resection candidates. Am J Respir Crit Care Med 1995:151:1472-80.

6. Yun $\mathbf{K H}$, Jeong $\mathbf{M H}, \mathrm{Oh} \mathrm{SK}$, et al. Preoperative plasma N-terminal pro-brain natriuretic peptide concentration and perioperative cardiovascular risk in elderly patients. Circ J 2008:72:195-9.

7. Rodseth RN, Padayachee L, Biccard BM. A meta-analysis of the utility of preoperative brain natriuretic peptide in predicting early and intermediate-term mortality and major adverse cardiac events in vascular surgical patients. Anaesthesia 2008;63:1226-33.
8. Ackland GL, Scollay JM, Parks RW, et al. Pre-operative high sensitivity C-reactive protein and postoperative outcome in patients undergoing elective orthopaedic surgery. Anaesthesia 2007;62:888-94.

9. Owens CD, Ridker PM, Belkin M, et al. Elevated C-reactive protein levels are associated with postoperative events in patients undergoing lower extremity vein bypass surgery. J Vasc Surg 2007;45:2-9.

10. Saklad M. Grading of patients for surgical procedures. Anesthesiology 1941:2:281-4.

11. American Society of Anesthesiologists. New classification of physical status. Anesthesiology 1963;24:111

12. Hall JC, Hall JL. ASA status and age predict adverse events after abdominal surgery. J Qual Clin Pract 1996;16:103-8.

13. Chijiiwa K, Yamaguchi K, Yamashita $\mathrm{H}$, et al. ASA physical status and age are no factors predicting morbidity, mortality, and survival after pancreatoduodenectomy. Am Surg 1996;62:701-5.

14. Brothers TE, Elliott BM, Robison J, et al. Stratification of mortality risk for renal artery surgery. Am Surg 1995:61:45-51.

15. Meixensberger $\mathbf{J}$, Meister $\mathrm{T}$, Janka $\mathbf{M}$, et al. Factors influencing morbidity and mortality after cranial meningioma surgery:a multivariate analysis. Acta Neurochir Supp/ 1996:65:99-101.

16. Hennein HA, Mendeloff EN, Cilley RE, et al. Predictors of postoperative outcome after general surgical procedures in patients with congenital heart disease. J Pediatr Surg 1994:29:866-70.

17. Houry S, Amenabar J, Rezvani A, et al. Should patients over 80 years old be operated on for colorectal or gastric cancer? Hepatogastroenterology 1994:41:521-5.

18. Karl RC, Schreiber R, Boulware D, et al. Factors affecting morbidity, mortality, and survival in patients undergoing Ivor Lewis esophagogastrectomy. Ann Surg 2000;231:635-43.

19. McCulloch P, Ward J, Tekkis PP. Mortality and morbidity in gastrooesophageal cancer surgery: initial results of ASCOT multicentre prospective cohort study. BM 2003;327:1192-7.

20. Prause G, Offner A, Ratzenhofer-Komenda B, et al. Comparison of two preoperative indices to predict perioperative mortality in non-cardiac thoracic surgery. Eur $J$ Cardiothorac Surg 1997;11:670-5.

21. Reid BC, Alberg AJ, Klassen AC, et al. The American Society of Anesthesiologists' class as a comorbidity index in a cohort of head and neck cancer surgical patients. Head Neck 2001;23:985-94

22. Michel JP, Klopfenstein C, Hoffmeyer P, et al. Hip fracture surgery: is the pre-operative American Society of Anesthesiologists (ASA) score a predictor of functional outcome? Aging Clin Exp Res 2002;14:389-94.

23. Pickering SA, Esberger D, Moran CG. The outcome following major trauma in the elderly. Predictors of survival. Injury 1999;30:703-6.

24. Wolters $\mathbf{U}$, Wolf $\mathrm{T}$, Stutzer $\mathrm{H}$, et al. ASA classification and perioperative variables as predictors of postoperative outcome. Br J Anaesth 1996;77:217-22.

25. Wolters U, Wolf $T$, Stutzer H, et al. Risk factors, complications, and outcome in surgery: a multivariate analysis. Eur J Surg 1997:163:563-8.

26. Peersman G, Laskin R, Davis J, et al. ASA physical status classification is not a good predictor of infection for total knee replacement and is influenced by the presence of comorbidities. Acta Orthopaedica Belgica 2008:74:360-4.

27. Goldman L, Caldera DL, Nussbaum SR, et al. Multifactorial index of cardiac risk in noncardiac surgical procedures. N Engl J Med 1977;297:845-50.

28. Eagle KA, Berger PB, Calkins $\mathrm{H}$, et al. ACC/AHA guideline update for perioperative cardiovascular evaluation for noncardiac surgery-executive summary a report of the American College of Cardiology/American Heart Association Task Force on Practice Guidelines (Committee to update the 1996 guidelines on perioperative cardiovascular evaluation for noncardiac surgery). Circulation 2002; 105:1257-67.

29. Poldermans D, Bax JJ, Boersma E, et al. Guidelines for pre-operative cardiac risk assessment and perioperative cardiac management in non-cardiac surgery The Task Force for Preoperative Cardiac Risk Assessment and Perioperative Cardiac Management in Non-cardiac Surgery of the European Society of Cardiology (ESC) and endorsed by the European Society of Anaesthesiology (ESA). Eur Heart $J$ 2009:30:2769-812

30. Lee TH, Marcantonio ER, Mangione CM, et al. Derivation and prospective validation of a simple index for prediction of cardiac risk of major noncardiac surgery. Circulation 1999:100:1043-9.

31. Ford MK, Beattie SW, Wijeysundera DN. systematic review: prediction of perioperative cardiac complications and mortality by the revised cardiac risk index. Ann Intern Med 2010;152:26-35.

32. Choi J-H, Cho DK, Song Y-B, et al. Preoperative NT-proBNP and CRP predict perioperative major cardiovascular events in non-cardiac surgery. Heart 2010;96:56-62.

33. Grocott MPW, Browne JP, Van der Meulen J, et al. The Postoperative Morbidity Survey was validated and used to describe morbidity after major surgery. J Clin Epidemiol 2007;60:919-28.

34. Shukri F, Khuri MD, Henderson WD, et al. Determinants of long-term survival after major surgery and the adverse effect of postoperative complications. Ann Surg 2005;242:326-43.

35. Charlson ME, Pompei P, Ales KL, et al. A new method of classifying prognostic comorbidity in longitudinal studies: development and validation. J Chronic Dis 1987; 40:373-83. 
36. Charlson M, Szatrowski TP, Peterson J, et al. Validation of a combined comorbidity index. J Clin Epidemiol 1994;47:1245-51.

37. Birim 0, Maat AP, Kappetein AP, et al. Validation of the Charlson comorbidity index in patients with operated primary nonsmall cell lung cancer. Eur J Cardiothorac Surg 2003;23:30-4.

38. Ouellette JR, Small DG, Termuhlen PM. Evaluation of Charlson-Age Comorbidity Index as predictor of morbidity and mortality in patients with colorectal carcinoma. J Gastrointest Surg 2004;8:1061-7.

39. Ghali WA, Hall RE, Rosen AK, et al. Searching for an improved clinical comorbidity index for use with ICD-9-CM administrative data. J Clin Epidemiol 1996:49:273-8.

40. Tao LS, Mackenzie CR, Charlson ME. Predictors of postoperative complications in the patient with diabetes mellitus. J Diabetes Complications 2008;22:24-8.

41. Wang CY, Lin YS, Tzao C, et al. Comparison of Charlson comorbidity index and Kaplan-Feinstein index in patients with stage I lung cancer after surgical resection. Eur J Cardiothorac Surg 2007;32:877-81.

42. Froehner M, Koch R, Litz R, et al. Comparison of the American Society of Anesthesiologists Physical Status classification with the Charlson score as predictors of survival after radical prostatectomy. Urology 2003;62:698-701.

43. Schroeder RA, Marroquin CE, Bute BP, et al. Predictive indices of morbidity and mortality after liver resection. Ann Surg 2006;243:373-9.

44. Copeland GP, Jones D, Walters M. POSSUM: a scoring system for surgical audit. $\mathrm{Br}$ J Surg 1991:78:355-60.

45. Sagar PM, Hartley MN, MacFie J, et al. Comparison of individual surgeon's performance. Risk-adjusted analysis with POSSUM scoring system. Dis Colon Rectum 1996;39:654-8.

46. Curran JE, Grounds RM. Ward versus intensive care management of high risk surgical patients. Br J Surg 1998:85:956-61.

47. Bennett-Guerrero E, Hyam JA, Shaefi S, et al. Comparison of P-POSSUM risk adjusted mortality rates after surgery between patients in the USA and the UK. Br J Surg 2003;90:1593-8.

48. Brooks MJ, Sutton R, Sarin S. Comparison of Surgical Risk Score, POSSUM and p-POSSUM in higher-risk surgical patients. Br J Surg 2005;92:1288-92.

49. Pratt W, Joseph S, Callery MP, et al. POSSUM accurately predicts morbidity for pancreatic resection. Surgery 2008;143:8-19.

50. Neary WD, Crow P, Foy C, et al. Comparison of POSSUM scoring and the Hardman Index in selection of patients for repair of ruptured abdominal aortic aneurysm. $\mathrm{Br} \mathrm{J}$ Surg 2003;90:421-5

51. Campillo-Soto A, Flores-Pastor B, Soria-Aledo V, et al. [The POSSUM scoring system: an instrument for measuring quality in surgical patients] (In Spanish). Cirugia Espanola 2006;80:395-9.

52. Tambyraja AL, Kumar S, Nixon SJ. POSSUM scoring for laparoscopic cholecystectomy in the elderly. ANZ J Surg 2005;75:550-2.

53. Bollschweiler $\mathbf{E}$, Lubke T, Monig SP, et al. Evaluation of POSSUM scoring system in patients with gastric cancer undergoing D2-gastrectomy. BMC Surg 2005:5:8.

54. Senagore AJ, Warmuth AJ, Delaney CP, et al. POSSUM, p-POSSUM, and Cr-POSSUM: implementation issues in a United States healthcare system for prediction of outcome for colon cancer resection. Dis Colon Rectum 2004:47:1435-41

55. Lam CM, Fan ST, Yuen AW, et al. Validation of POSSUM scoring systems for audit of major hepatectomy. Br J Surg 2004;91:450-4.

56. Tekkis PP, Kessaris N, Kocher HM, et al. Evaluation of POSSUM and P-POSSUM scoring systems in patients undergoing colorectal surgery. [see comment]. Br J Surg 2003;90:340-5

57. Wijesinghe LD, Mahmood T, Scott DJ, et al. Comparison of POSSUM and the Portsmouth predictor equation for predicting death following vascular surgery. [see comment]. Br J Surg 1998;85:209-12.

58. Whiteley MS, Prytherch DR, Higgins B, et al. An evaluation of the POSSUM surgical scoring system. Br J Surg 1996;83:812-15.

59. Prytherch DR, Whiteley MS, Higgins B, et al. POSSUM and Portsmouth POSSUM for predicting mortality. Physiological and Operative Severity Score for the enUmeration of Mortality and morbidity. Br J Surg 1998;85:1217-20.
60. Ramesh VJ, Rao GS, Guha A, et al. Evaluation of POSSUM and P-POSSUM scoring systems for predicting the mortality in elective neurosurgical patients. $\mathrm{Br} \mathrm{J}$ Neurosurg 2008;22:275-8.

61. Tamijmarane A, Bhati CS, Mirza DF, et al. Application of Portsmouth modification of physiological and operative severity scoring system for enumeration of morbidity and mortality (P-POSSUM) in pancreatic surgery. World J Surg Oncol 2008;6:39.

62. Lai F, Kwan TL, Yuen WC, et al. Evaluation of various POSSUM models for predicting mortality in patients undergoing elective oesophagectomy for carcinoma. [see comment]. Br J Surg 2007:94:1172-8.

63. Vather R, Zargar-Shoshtari K, Adegbola S, et al. Comparison of the possum, P-POSSUM and Cr-POSSUM scoring systems as predictors of postoperative mortality in patients undergoing major colorectal surgery. ANZ J Surg 2006;76:812-16.

64. Gocmen E, Koc M, Tez M, et al. Evaluation of P-POSSUM and 0-POSSUM scores in patients with gastric cancer undergoing resection. Hepato-Gastroenterology 2004;51:1864-6.

65. Horzic M, Kopljar M, Cupurdija K, et al. Comparison of P-POSSUM and Cr-POSSUM scores in patients undergoing colorectal cancer resection. Arch Surg 2007; 142:1043-8.

66. Tekkis PP, Prytherch DR, Kocher HM, et al. Development of a dedicated riskadjustment scoring system for colorectal surgery (colorectal POSSUM). Br J Surg 2004:91:1174-82

67. Dutta S, Horgan PG, McMillan DC. POSSUM and its related models as predictors of postoperative mortality and morbidity in patients undergoing surgery for gastrooesophageal cancer: a systematic review. World J Surg 2010;34:2076-82.

68. Prytherch DR, Sirl JS, Weaver PC, et al. Towards a national clinical minimum data set for general surgery. Br J Surg 2003;90:1300-5.

69. Nichols KG, Prytherch DR, Fancourt MF, et al. Risk-adjusted general surgical audit in octogenarians. ANZ J Surg 2008:78:990-4.

70. Prytherch DR, Ridler BMF, Ashley S. Risk-adjusted predictive models of mortality after index arterial operations using a minimal data set. Br J Surg 2005;92:714-18.

71. Apgar V. A proposal for a new method of evaluation of the newborn infant. Curr Res Anesth Analg 1953;32:260-7.

72. Gawande AA, Kwaan MR, Regenbogen SE, et al. An Apgar score for surgery. J Am Coll Surg 2007:204:201-8.

73. Regenbogen SE, Ehrenfeld JM, Lipsitz SR, et al. Utility of the Surgical Apgar Score Validation in 4119 Patients. Arch Surg 2009:144:30-6.

74. Khuri SF, Daley J, Henderson WG, et al. The National Veterans Administration Surgical Risk Study: risk adjustment for the comparative assessment of the quality of surgical care. J Am Coll Surg 1995;180:519-31.

75. Cohen ME, Bilimoria KY, Ko CY, et al. Development of an American College of Surgeons National Surgery Quality Improvement Program: Morbidity and Mortality Risk Calculator for Colorectal Surgery. J Am Coll Surg 2009;208:1009-16.

76. Grocott MPW, Levett DZH, Matejowsky C, et al. ASA scores in the preoperative patient: feedback to clinicians can improve data quality. J Eval Clin Pract 2007:13:318-19.

77. Dindo D, Hahnloser D, Clavien PA. Quality assessment in surgery: riding a lame horse. Ann Surg 2010;251:766-71.

78. Hutter MM, Rowell KS, Devaney LA, et al. Identification of surgical complications and deaths: an assessment of the traditional surgical morbidity and mortality conference compared with the American College of Surgeons-National Surgical Quality Improvement Program. J Am Coll Surg 2006;203:618-24.

\section{ANSWERS}

1. $A(T) ; B(F) ; C(F) ; D(T) ; E(F)$

2. $A(T) ; B(F) ; C(T) ; D(F) ; E(T)$

3. $A(T)$ : $B(F)$ : $C(F)$ : $D(F) ; E(T)$

4. $A(F) ; B(T) ; C(F) ; D(F) ; F(T)$

5. $A(T) ; B(T) ; C(F) ; D(T) ; E(T)$ 